Cristiani.-Experimental Infective Neothyroiditis. " Revue Méd. de la Suisse Romande," June, 1903.

In earlier papers Cristiani reported his experiments on the transplanting of thyroid grafts, and showed that such grafts became active functioning thyroid glands. The present paper records his observations on the powers of such grafts to withstand or recover from various inflammatory processes.

The animals used were rabbits, guinea-pigs, and white rats. The grafts were placed in pockets formed between the skin and cartilage of the lobe of the ear, and allowed to remain various periods, from a few days to several months, before being submitted to experiment, so that some were only beginning to become revascularized, others were already active glands-" neothyroid glands." In one series of experiments cultures of streptococci from various sources were injected into and around the grafts. All became more or less inflamed; three suppurated; but on microscopic examination some months later, active thyroid tissue was found in all but one case, and it was a graft only some nine days old when the experiment was made. A second series of experiments was carried out with staphylococcus, coli bacillus, and bacillus pyocyaneus. After recovering from the more or less severe inflammation set up by the injections, active gland tissue was found in five out of seven cases; and in the two in which the gland tissue was destroyed by the inflammation the grafts were very young (three and seven days). Again, other " neothyroids" were burned with the thermo-cautery, others injected with irritant liquids, with results similar to the foregoing.

These experiments therefore prove that thyroid grafts transplanted according to Cristiani's method grow to form glands which are stable and active, and are also capable of resisting and recovering from serious inflammatory lesions. Arthur J. Hutchison.

Wilson, R. A.-Lodgment of a Foreign Body in the Air-Passages for Seventy-two Days. " "Lancet," November 22, 1902.

The patient was a man aged forty-five, who was thrown from a cart while intoxicated. He imagined that he had broken his ribs. No bony crepitus could be elicited. His temperature was $100^{\circ}$, his pulse 84 , and his respirations were 24 per minute. There was dulness, and increase of vocal fremitus on the right infrascapular region. $\mathrm{He}$ remained in bed a week, and then returned to work. While suffering from one of his attacks of dyspnoea and cough, he expectorated the proximal portion of a vulcanite pipe-stem half an inch long, which he recognised as belonging to the pipe he was smoking at the time of the accident. A few days later all subjective symptoms and physical sigus had disappeared.

StClair Thomson.

\title{
EAR.
}

Brunard and Labarre, E.-A Case of Typhoid Fever complicated b!y Mastoiditis and Cerebral Abscess; Two Operations; Death: Autopsy. "La Presse Oto-Laryngologique Belge," March, 1902.

A girl, aged seven, passed through a severe attack of enteric fever. In the fourth week, when defervescence was complete, a succession of abscesses containing staphylococci began to form in the scalp. A fort- 
night later, while this was still going on, a tender swelling appeared in front of the left ear and puffiness of the mastoid, preceded by a slight discharge of pus from the meatus. The mastoid operation was performed without delay; the antrum was full of granulations without pus. Afterwards there was slight fever for some days, then the temperature fell to normal. The wound looked well, and was cicatrizing in a healthy manner, and there was no reason to suspect intracranial mischief. Twenty-four days after the operation serious symptoms came on suddenly-namely, drowsiness, apathy, slowness and irregularity of the pulse, bilious vomiting, and slight ptosis of the left eyelid. Taches cerebrales appeared slowly. The abdomen was soft and not retracted. The following day the child had numerous general convulsions. There was optic neuritis; the left pupil was contracted, the movements of the eye normal. No stiffness of the neck was observed. The pulse was very irregular and slow.

A further operation was performed the same afternoon, by which time the patient was quite comatose, with a temperature of $95.5^{\circ} \mathrm{F}$. After the removal of some granulations, a fistulous opening, from which flowed some drops of pus, was found in the roof of the antrum. The middle fossa of the skull was then opened through the squamous portion. The meninges, normal in colour, were strongly bulging outwards and without pulsation. Stacke's protector, introduced at the level of the seat of the fistula, liberated a large quantity of pus. As it flowed away the pulsations of the brain returned. The operation was terminated by irrigation with sterilized serum and the introduction of a drain of iodoform gauze.

The immediate results of the operation were excellent. The pulse quickly rose to 80 , and the child soon regained consciousness. She passed a good night, and the convulsions and sickness ceased. When the wound was dressed, the day after the operation, 50 grammes of thick green pus came away. The pulse was still irregular. On the third day the child was lively, and took food well. At midnight, however, she relapsed into coma, and all the signs of basic meningitis appeared, which soon proved fatal.

At the autopsy, besides purulent basic meningitis, a large abscess cavity, 7 centimetres long, was found in the left hemisphere.

Chichele Nourse.

Gaudier.-Two Cases of Cerebellar Abscess of Otitic Origin. "L'Echo Méd. du Nord," May 31, 1903.

The first case was a boy, aged twelve, with otorrhca and mastoid fistula of the left ear of two years' duration. When he was admitted to hospital he presented no signs of intracranial mischief, but during the night after admission he was suddenly seized with vomiting, vertigo, stiffness of neck and violent occipital headache, followed by coma; temperature in rectum was $36 \cdot 2^{\circ}$, pulse between 45 and 50 . The mastoid operation was performed, the lateral sinus was exposed and punctured, but it yielded only pure blood. The mastoid cavity was then enlarged upwards and forwards, and the dura mater of the middle fossa exposed freely. The dura bulged and did not pulsate. Explora. tion of temporo-sphenoidal lobe was negative. Owing to the condition of the child, the operation had to be stopped without opening the cerebellar fossa. The coma passed off, and next day the pulse was 55, and temperature $363^{\circ}$. This improved condition lasted till the following 
evening, when suddenly the child became convulsed, and then comatose, and died.

Post-mortem.-A large superficial antero-external abscess of left side of cerebellum; no meningitis; no cerebral abscess.

The second case was a boy, aged eighteen, with intermittent otorrhcea of old standing. For three days his condition had been the following: Sudden onset of nausea, then vomiting, vertigo, and disturbance of equilibrium, and severe left-sided headache, most marked at a point 2 centimetres behind the mastoid process. This condition was followed by prostration and coma; no swelling over the mastoid; slight discharge from ear continued; a perforation in the antero-inferior quadrant of the membrane, but no pulsation and no granulation tissue. Pulse, 45 ; rectal temperature, $36^{\circ}$. The skull was trephined at once over the cerebellum. As soon as the bone was removed very fœetid pus appeared, and an irregular hole in the dura was found, through which the point of a tinger could be passed into the cerebellum for more than a centimetre. The cavity was then washed out with peroxide of hydrogen, and drainage secured by a strip of gauze. The patient regained consciousness, the pulse rose to 72 and temperature to $37.3^{\circ}$ on the day of the operation, and the headache disappeared. Convalescence practically uneventful. A fortnight after the operation the mastoid was operated on. It was much sclerosed; some granulations were found in the antrum; the lateral sinus was healthy.

Gaudier calls special attention to the localization of the head pain in the second case, which he considers of great value in diagnosing cerebellar from cerebral abscess.

In the absence of special indications, he recommends opening the cranium through the mastoid. If pathological conditions are found in the bone, their situation will guide the operator to the intracranial lesion. In the absence of such guidance, if there are any localizing signs pointing to abscess in the sphenoidal or occipital lobes, of course these regions should be explored first; on the other hand, if, besides the general signs, there are bilious vomiting and stiffness of the neck, the cerebellum should be first explored; finally, having failed to find pus in the one region, the other should always be explored. Had this been done in Case I. the patient's life might have been saved.

\section{Arthur.J. Hutchison.}

Gillot, V. (Algiers).-Cerebellar Abscess of Otic Origin. "La Presse Oto-Laryngologique Belge," September, 1902.

An Arab, aged twenty-three, who had only been ill a few days, was admitted to the hospital with all the signs of cerebellar trouble. $\mathrm{He}$ had severe and continuous occipital headache, staggering gait, inability to stand upright without falling, vertigo even when in complete repose, and incessant vomiting. Slight nystagmus was observed. The tendon reflexes were much more marked in the right arm and leg than in the left. There were no paralyses, contractions, nor disturbances of sensation. There was no fever, nor any discharge from the ears or mastoid tenderness. A cerebellar tumour was suspected. Death ensued after fifteen days. At the autopsy an abscess containing thick yellow pus was found in the white substance of the right lobe of the cerebellum; close to it, situated in the gray matter, was a smaller one. The meninges were adherent to the upper border of the petrous bone, where there was a minute point of caries.

An examination of the bone showed the existence of mastoiditis on 
the same side. The cortex, which was very thick and hard, prevented the pus from finding an exit outwardly. The author thinks that influenza, which was epidemic in Algiers at the time, was the cause of the trouble.

Chichele Nourse.

Hinsberg, V. (Breslau).- Suppurations in the Labyrinth. "Arch. of Otol.," vol. xxxi., Nos. 2 and 3.

The writer considers that a labyrinth infection from the middle ear is only possible through a perforation of the labyrinth wall. This may be traumatic, as from fracture or operation, the latter either accidental or intentional. Meningitis following unskilful attempts at extraction of foreign bodies is mostly due to perforation of the labyrinth wall. During the "radical" operation the external semicircular canal may be opened or the stapes may be dislocated. The latter is more dangerous than the former. Nore frequently labyrinth suppuration occurs as the result of the destructive action of middle-ear suppuration, and this is more common than has been supposed. Koerner now includes in his tables a division, "labyrinth suppurations following suppurations of the middle ear," with three subdivisions, "empyema of the endolymphatic sac," " empyema of the vestibule," and "operations on the labyrinth."

In sixty-one cases the site of entrance of the infection could be identified:

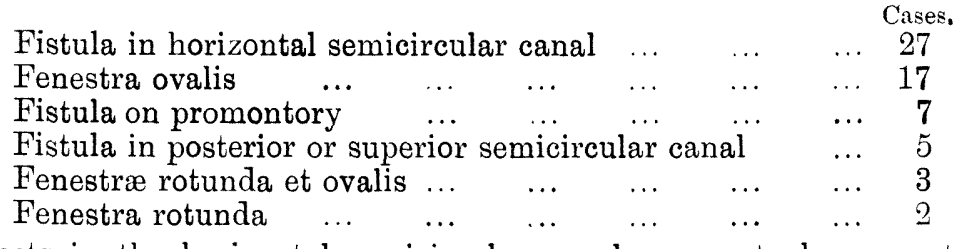

Defects in the horizontal semicircular canal appear to be present in from 1.06 to 1.2 per cent. of cases of chronic middle-ear suppuration. The vertical canals are occasionally infected.

The middle-ear disease was in 89 of the author's cases acute in 18 , chronic in 71 , of which 21 were complicated with cholesteatoma. Jansen found cholesteatoma in 71 cases, tuberculosis in 16 .

As regards the pathological anatomy, the labyrinth may be infected in its entirety, and be filled with pus or infiltrated with round-celled tissue, which may become organized and ossify. In some cases the whole frame of the cochlea may become detached and exfoliated. The suppuration is at times confined to a portion of the labyrinth. Extension into the cranial cavity usually follows the normal channels, such as the aqueducts or the internal auditory meatus, but sometimes a dehiscence is present, congenital or acquired. Extradural abscess may result, especially, according to Jansen, in neglected acute cases. The jugular bulb may be infected through the veins of the aqueducts. The pathways from the labyrinth lead into the posterior cranial fossa, except when the anterior crus of the superior semicircular canal is involved and the middle fossa is thus reached. Encephalic abscess of labyrinthine origin is, so far as is known, always cerebellar.

The symptoms are very inconstant. Fever may be present, but in many cases it is absent. Absolute or practical deafness on the affected side is present in most cases, but in some a considerable amount of hearing-power has been observed, the tuning-fork on the vertex even being better heard in the affected ear. In this case the cochlea is probably unaffected. Vertigo or instability of gait ("duck-gait") is 
usual, but sometimes this symptom is wanting, most probably in cases of very slow destruction. Nystagmus is very frequent, and Jansen found it in every case of traumatic perforation of the horizontal canal. Among other symptoms have been noted nausea, vomiting, and headache.

The course frequently terminates fatally, especially from meningitis, but recovery with permanent deafness sometimes ensues.

Intracranial complications are frequent, and occurred 108 times in 198 cases collected by the author. The pathway in 43 was as follows:

Internal meatus

Internal meatus and aqueducts

Aqueductus vestibuli...

- Aqueductus cochleæ ...

Fistulæ in vertical canals

$$
\begin{array}{ccccc} 
& & & \multicolumn{2}{r}{\text { Cases. }} \\
\ldots & \ldots & \ldots & \ldots & 25 \\
\ldots & \ldots & \ldots & \ldots & 1 \\
\ldots & \ldots & \ldots & \ldots & 8 \\
\ldots & \ldots & \ldots & \ldots & 1 \\
\ldots & \ldots & \ldots & \ldots & 8
\end{array}
$$

The following were the terminations:

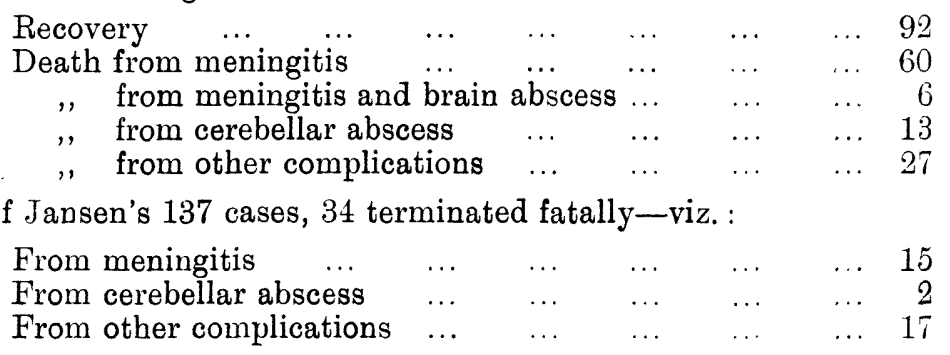

These figures show that meningitis is by far the most frequent complication.

In a case of Brieger's relief was afforded by lumbar puncture; recovery took place, and Brieger attributed the symptoms to a localized meningitis. The influence of operations on the middle ear in the course of labyrinthine suppuration seems to vary. In some cases they suffice to allay the inflammation in the labyrinth, but in other cases labyrinthine irritation seems to be more intense after the operation.

The diagnosis is fairly easy in cases where vertigo, deafness, nystagmus, nausea, and headache are well developed, but from what has been said above, it will be obvious that even in dangerous cases there may be no guiding symptoms; thus, deafness may be absent when the suppuration is confined to the vestibule and semicircular canals, and the symptoms may be then practically identical with those of cerebellar abscess, though in the latter there will be more marked emaciation, prostration, mental changes, optic neuritis, slowness of pulse, and staggering gait. The affections may exist together, as cerebellar abscess is frequently the result of labyrinthine suppuration; exploratory operations may be the only means of diagnosing.

The disease is not necessarily fatal, and operation on the labyrinth, simultaneous with, or subsequent to the radical mastoid operation, may be successful. The writer has collected reports of 31 operations on the labyrinth; out of these 23 recovered, 6 died, and in 2 the termination is not mentioned; the results are, therefore, on the whole, rather encouraging than otherwise; the chief danger seems to be the concussion from chiselling and the tearing of protective 
adhesions. The writer quotes the indications for operation on the labyrinth as formulated by Jansen, to the effect that in all cases with severe labyrinthine symptoms opening of the vestibule is allowed, and in those cases in which the conditions found in the middle ear on radical operation do not sufficiently account for those severe symptoms, the opening of the vestibule is necessary; when the labyrinthine symptoms, though present, are less severe, but a labyrinthine fistula is present and the focus of retained pus is not found in or on the mastoid bone, the vestibule should be opened; this should also be done in cases with distinct labyrinth symptoms where the presence of cerebellar or large extradural abscess is probable; also in cases of fistula in the semicircular canals with symptoms of meningeal irritation. Hinsberg adds to these that in all cases which call for operation on the vestibule the cochlea should also be opened. He points out, further, the difficulty of determining the seriousness of a diagnosed labyrinthine affection, in riew of the fact that cases of an apparently serious character may heal up spontaneously, and that, on the other hand, cases in which the symptoms are not at all severe have suddenly developed meningitis. With regard to the operation on the labyrinth, the following are the steps described:

1. Radical operation with removal of the posterior wall of the meatus, sufticient to expose the region of the oval window.

2. Opening of the foramen ovale and, if opening of the cochlea is intended, removal of the promontory.

3. Introduction of a probe through the oval window and, under its suidance, opening of the vestibule from the horizontal canal, if possible along its anterior crus, or, under difficult anatomical conditions, from behind.

A very fine electric burr is recommended for the purpose.

[This extension of operative interference adds to the responsibility as well as to the usefulness of the aural surgeon. Hinsberg's original paper, with the bibliography, will be invaluable to the student of the subject. A case of radical mastoid operation in which Mr. Ballance followed the disease into the labyrinth, the semicircular canals being in part destroyed and the back of the vestibule opened, and subsequently practised epithelial grafting with excellent results, was brought by him before the Otological Society (Journal of LaRYngology, June, 1900, p. 314).-D. G.]

Dundas Grant.

Knapp, Arnold, and Jordan, C. H. R.-A Brief Report of Forty Radical Operations for Chronic Purulent Otitis and Complications, with Remarks. "Arch. of Otol.," vol. xxxii., No. 2.

These were grouped as: (1) Cases of simple caries; (2) cases of caries with severe symptoms, consisting in headache and nausea, in five of which there was defect of the tegmen antri; (3) cases of acute reinfection of the antrum ; (4) cases with fistula in the labyrinth ; (5) cas $€ \mathrm{~S}$ of intracranial complication. The group with labyrinthine fistula is particularly interesting, the classical symptoms being vertigo and nystagmus, the former increasing when the head is turned towards the affected side, nystagmus becoming more distinct when the eyes are turned in the opposite direction. Skin-grafting was practised four times according to Ballance's method: twice grafts were secondarily inserted through the meatus, and twice an immediate skin-graft was placed over the facial spur and promontory wall. Dundas Grant. 
Körte (Berlin).-Case of Implantation of the Facial to the Hypoglossal Nerve. "Deutsche Med. Woch.," 1903, No. 17.

The facial nerve was divided close to the stylo-mastoid foramen during a resection of the petrous bone. The distal end was then (for reasons we need not detail) attached to the hypoglossal nerve, which, as the result of the destruction of the posterior belly of the digastric, was more accessible than the spinal accessory ; there resulted a marked paralysis in the area supplied by both nerves, with the complete reaction of degeneration. Half a year later, electrical excitability and active movements slowly returned; but when the muscles of the face were moved, the tongue moved at the same time, and vice versa. The writer would deprecate implantation into the hypoglossal nerve mainly on account of the temporary hypoglossal paralysis, which, combined with paralysis of the buccinator resulting from the lesion of the facial nerve, produced a very serious defect in chewing and deglutition; he therefore recommends, in preference, the selection of the spinal accessory nerve for the purpose, as paresis of the sterno-mastoid and trapezius is much less annoying than that of the lingual muscles.

Dundas Grant.

Muck (Rostock).-Entotic Murmur due to Aneurism of the Occipital Artery. "Arch. of Otol.," vol. xxxii., No. 2.

The patient, about eight years after an injury to the back of her head, followed by unconsciousness, began to observe a continuous pulsatile beating in her ear. There was found a lobular, pulsating tumour situated under the skin, beginning at the posterior margin of the mastoid process, and extending backwards to within 2 or 3 centimetres of the occipital protuberance. Pulsation was checked by pressure exerted at its anterior lower corner. Dr. Muck dissected away this aneurismal sac with considerable difficulty. The pulsation was then felt over an area of 2 centimetres in diameter, but the subjective aural symptoms had completely disappeared.

Dundas Grant.

Piff (Prag). - The Operative Exposure of the Bulb of the Internal Jugular Vein. "Arch. für Ohrenheilkunde," Narch, 1903.

In cases in which the exposure by Grunert's method seemed impossible Piffl succeeded by chipping away the lower part of the osseous meatus and floor of the tympanum. Access is gained by carrying the ordinary mastoid incision downwards and turning the soft parts forwards, then detaching the periosteum from the tympanic bone by means of blunt elevators. The styloid process may, if necessary, be freed from the soft parts attached to it and removed.

Dundas Grant.

Schiffers, F.-Chronic Suppuration of the Middlc Ear; Thrombosis of the Cavernous Sinus. "La Presse Oto-Laryngologique Belge," March, 1902.

The patient, aged twenty-two, was the subject of an old-standing suppuration of the left ear. On January 24,1893 , during his term of military service, he applied to a hospital, and was admitted on the medical side. He had intense headache, violent pain in the left ear, pains in the limbs, and insomnia. The same evening he had a rigor lasting twenty minutes. On February 2 the pain in the ear was much worse. The patient became delirious. The purulent discharge from the ear was abundant and very foetid. The next day coma supervened. 
The head and eyes were slightly turned away from the left; there was ptosis of the left eyelid and exophthalmos with odema. The sixth nerve was not affected. The left pupil was widely dilated, and did not react to light. The condition of the patient remained the same until February 7, when he appeared a little better; but he did not become conscious until February 11. The motor oculi was completely paralyzed. An ophthalmoscopic examination on March 10 showed a choked disc on the left side.

From January $2 \pm$ to March 2 the temperature oscillated irregularly between $98 \cdot 6^{\circ} \mathrm{F}$. and $104^{\circ} \mathrm{F}$. After the latter date it became normal ; at the same time the condition of the patient improved. The treatment consisted of electricity, hypodermic injections of strychnine, and for the ear merely antiseptic dressings.

The patient left the hospital on June 15. He considered himself cured, and was pronounced fit to resume military duties, which he did, without troubling himself in the least about his ear. Nearly a year later, on May 21, 1894, he came to the clinique of otology with all the signs of acute meningitis, of which he died four days later. In the presence of those signs operation was out of the question.

At the autopsy an extra-dural collection of pus was found on the posterior surface of the petrous bone. The dura opposite this focus was mortified, and of a greenish colour. There were signs of former inflammation of the cavernous sinus-namely, adhesion of the wall to the bone, and organization of the thrombus. There was fibrino-purulent meningitis at the base of the brain.

Chichele Nourse.

Simon, R. M. (Birmingham).- - Case of Acute Meningitis and Cerebellar. Abscess. " Birmingham Med. Review," May, 1903.

The patient, a healthy girl, aged eighteen, had had discharge from both ears from early childhood, which had ceased for the previous five months. Great pain in the head, with temperature $97^{\circ} \mathrm{F}$; pulse 50 ; pupils dilated, face flushed. Seen on March 10; died on March 13 ; temperature rose to $100^{\circ} \mathrm{F}$. just before death.

Post-mortem examination revealed pus in left middle ear, abscess in left lobe of cerebellum, pointing outwards on the lateral border between the inferior and superior surfaces. Pus in the dura and beneath it along the longitudinal fissure. Pus from the abscess cavity showed abundant colonies of pneumococcus, a few staphylococci and streptococci. There was a doubtful history of influenza a fortnight before she was seen, and the pneumococcus is thought to have stirred up the old otitis media and reinfected the region. Barclay J. Baron.

\section{THERAPEUTICS.}

Cairns, D. Louis.-On the Treatment of Diphtheria by the Intracenous Injectron of Antidiphtheritic Serum. "Lancet," December 20, 1902.

The writer thought that the mortality from diphtheria, much reduced as it has been, might be further curtailed by (1) the exhibition of larger doses than those generally recommended, and (2) by the intravenous use of the remedy in certain cases. A number of cases are quoted to support these views. The dose employed subcutaneously varied from 4,000 to 20,000 units, and intravenously from 20,000 to 